\title{
Comprehensive bioinformatics analyses identified Homeobox B9 as a potential prognostic biomarker and therapeutic target for gastric cancer
}

\author{
Xiaofei Li ${ }^{1 \#}$, Shujia Chen ${ }^{1 \#}$, Yinghui Zhu ${ }^{1}$, Jiayue Fei ${ }^{1}$, Liaoyuan Song', Guoyan Sun ${ }^{1}$, Wei Niu ${ }^{1}$, \\ Lianyi Guo', Jiwei Wang ${ }^{2}$ \\ ${ }^{1}$ Department of Gastroenterology, The First Affiliated Hospital of Jinzhou Medical University, Jinzhou, China; ${ }^{2}$ Department of Gastrointestinal \\ Surgery, Xuzhou Central Hospital, Xuzhou, China \\ Contributions: (I) Conception and design: X Li; (II) Administrative support: J Wang; (III) Provision of study materials or patients: S Chen; (IV) \\ Collection and assembly of data: L Guo; (V) Data analysis and interpretation: Y Zhu; (VI) Manuscript writing: All authors; (VII) Final approval of \\ manuscript: All authors. \\ "These authors contributed equally to this work. \\ Correspondence to: Jiwei Wang. Department of Gastrointestinal Surgery, Xuzhou Central Hospital, Xuzhou 221009, China. \\ Email: zxyywv82847@126.com; Lianyi Guo. Department of Gastroenterology, The First Affiliated Hospital of Jinzhou Medical University, Jinzhou \\ 121001, China. Email: angel_gly@163.com.
}

Background: The Homeobox B $(H O X B)$ family promotes tumor progression, but the mechanism of its action in gastric cancer (GC) is unclear. We sought to identify the HOXB family members that are critical to the prognosis of GC patients.

Methods: The Oncomine, Gene Expression Profiling Interactive Analysis (GEPIA), cBioPortal, UALCAN, Kaplan-Meier plotter, and the GeneMANIA databases were used to analyze the messenger RNA (mRNA) expression levels, prognostic value, and gene-gene interaction network of the HOXB9 family members in GC. The expression of HOXB9 in GC and its relationship with various clinicopathological parameters and the prognosis of patients were verified by immunohistochemistry.

Results: The expression of $H O X B 3, H O X B 5, H O X B 6, H O X B 7, H O X B 9$, and HOXB13 mRNA was significantly upregulated in GC. There was a significant correlation between the upregulation of $H O X B 3$, $H O X B 5$, and $H O X B 9 \mathrm{mRNA}$ and a low overall survival (OS) rate. The high expression of $H O X B$, $H O X B$, and $H O X B 13$ mRNA was closely correlated to tumor grade and stage. $H O X B 9$ was the $H O X B$ family member most closely related to the occurrence and development of GC. A further analysis showed that HOXB 9 might be involved in deoxyribonucleic acid repair and division regulation. A validation study showed that the advanced cancer group had a higher level of $H O X B 9$ expression than the early cancer group. The high expression of $H O X B 9$ in gastric tissue plays an important role in the survival and prognosis of GC patients.

Conclusions: HOXB family members have different degrees of abnormal expression in GC. High HOXB9 expression in GC tissues was significantly correlated with a worse prognosis. Thus, HOXB9 is a potential novel biomarker and therapeutic target for GC.

Keywords: Gastric cancer (GC); Homeobox B9 (HOXB9); Homeobox B family (HOXB family); prognosis biomarker; therapeutic target

Submitted Aug 30, 2021. Accepted for publication Oct 16, 2021.

doi: 10.21037/jgo-21-598

View this article at: https://dx.doi.org/10.21037/jgo-21-598 


\section{Introduction}

Gastric cancer (GC) is characterized by high morbidity and mortality, and is the 5 th most common cancer type and the $3 \mathrm{rd}$ most common cause of cancer-related death worldwide (1). Great progress has been made in the surgical treatment and chemotherapy of GC; however, due to the recurrence and metastasis of GC, the 5-year survival rate of GC patients is still less than $20 \%(2,3)$. Additionally, the early diagnosis of GC remains a clinical challenge, as many patients are asymptomatic, the early lesions can be nonspecific, and there is a lack of effective biomarkers. Previous research has focused on the potential pathogenesis of GC and identifying novel biomarkers and therapeutic targets. However, the mortality rate of GC patients remains very high (4). Thus, research urgently needs to be conducted to examine the mechanisms underlying the occurrence and development of GC and identify highly sensitive and specific tumor biomarkers.

The Homeobox (HOX) gene family encodes a variety of homeodomain transcription factors that play an important role in embryonic development by regulating cell proliferation and differentiation (5). It has been confirmed that there are 39 Hox genes in mammals that are composed of 4 homologous clusters on the autosomal chromosomes $(6,7)$. The Homeobox B $(H O X B)$ family is critical for cell morphogenesis and differentiation $(8,9)$. The expressions of HOXB family members, including $H O X B 1$, НОХВ2, НОХВ3, НОХВ 4, НОХВ5, НОХВ6, НОХВ 7, $H O X B 8, H O X B 9$, and $H O X B 13$, have been confirmed to be associated with the progression of a variety of tumors. Specifically, HOXB1 has been shown to be downregulated in gliomas and lung cancers, and its expression inhibits the apoptosis and promotes the proliferation of cancer cells $(10,11)$. Additionally, HOXB2 has been shown to be upregulated in cervical cancer and pancreatic cancer $(12,13)$, and was found to be the targets of multiple small RNAs in colorectal cancer (CRC), glioblastoma, and acute myeloid leukemia (14-16). HOXB3 has been shown to be a target of microRNA-10a (miR-10a) and regulated by retinoic acid receptor signaling pathway in pancreatic cancer metastasis (17). HOXB4 is an important transcription factor involved in the progression of many cancer types, including lung, breast, prostate, and bladder cancer, and promotes cancer cell proliferation through the STAT3 pathway $(18,19)$. As an important factor in the development of the enteric nervous system, HOXB 5 has been shown to play a role in breast cancer biology by modifying intussusceptive angiogenesis (20). HOXB6 is highly expressed in hematopoietic stem/progenitor cells, and is downregulated during terminal differentiation, which suggests that its aberrant expression is implicated in hematological malignancies (21). As an important transcription factor, HOXB7 regulates many cancer cells' functions, including proliferation, invasion, migration, angiogenesis, and epithelial-mesenchymal transition (EMT) (22). The upregulated expression of HOXB8 has been observed in all stages of CRC (23), while a LINC01006/miR-2682-5p/HOXB8 feedback loop has been shown to promote cell growth and metastasis in prostate cancer (PCa) (24). HOXB9 has been shown to be highly expressed in $43 \%$ of breast cancer tissues and positively correlated with tumor grade (25). The abnormal expression of $H O X B 9$ has also been shown to indicate poor prognosis and promote tumor progression in patients with many other cancer types (26,27). Many studies have identified HOXB13 as a candidate tumor suppressor gene in several types of cancer, including CRC (28), melanoma (29), and breast cancer (30).

In $\mathrm{GC}$, the $H O X B$ factors have been shown to play critical roles in disease progression. The relationship between the abnormal expression of $H O X B$ genes and clinicopathological features and prognosis of GC has been reported. For example, previous studies have shown that HOXB3 and HOXB9 are associated with the development and prognosis of GC (31). Recent studies have shown that $H O X B 5$ may be an important regulator of the Wnt/ $\beta$-catenin signaling pathway and involved in the progression and metastasis of GC (32). HOXB8 plays an important role in the occurrence and metastasis of GC (33). The low expression of $H O X B 13$ promotes the occurrence, invasion and progression of GC, and is a poor prognostic factor of GC (34). However, systematic bioinformatics analyses on the expressions of $H O X B$ factors in tumors and their roles in GC prognosis are lacking.

In this study, using published databases, we comprehensively analyzed the expression patterns of different HOXBs in normal tissues and the tumor tissues of GC patients, and determined their potential functions and prognostic value in GC. We used several online analysis tools to analyze and evaluate the correlation between HOXB9 expression, prognosis and clinicopathological factors in patients with GC, which was further verified by immunohistochemistry. In addition, using a new cohort of GC patients at our hospital, we confirmed that HOXB9 is a potential biomarker that can be used to diagnose and 
predict the prognosis of GC. However, this was different from previous studies. The results of Chang et al. (34) study suggested that $H O X B 9$ was a tumor suppressor in gastric carcinoma, and its activity was controlled by different regulatory mechanisms such as the hexapeptide motif as a "brake" in that case. The results of these regulatory effects could lead to either oncogenic or tumor suppressive roles of $H O X B 9$, depending on the context of the particular type of cancer involved (35). We present the following article in accordance with the REMARK reporting checklist (available at https://dx.doi.org/10.21037/jgo-21-598).

\section{Methods}

\section{Ethics statement}

From 2010 to 2014, a total of 70 paraffin sections of GC tissues were selected from The First Affiliated Hospital of Jinzhou Medical University. Written informed consent was obtained from all the participants. This study was approved by the Ethics Committee of The First Affiliated Hospital of Jinzhou Medical University (No. 1900034790). All procedures performed in this study involving human participants were in accordance with the Declaration of Helsinki (as revised in 2013). None of the patients in the analysis received radiotherapy or chemotherapy before surgery. Clinical data were collected for all patients.

\section{Bioinformatics analyses of gene expressions}

Using the Oncomine gene expression array data set (https:// www.oncomine.org/resource/login.html), we analyzed the expression of $H O B X$ transcriptome data among different cancers. There were 86,733 normal tissue specimens and tumor tissue specimens (36). The Student's $t$-test was used, and the $\mathrm{P}$ value and fold change value were set to $1 \mathrm{E}-4$ and 2 , respectively.

The Gene Expression Profiling Interactive Analysis (GEPIA; http://gepia.cancer-pku.cn/) online database was used to analyze the expression of HOXBs in GC and normal tissues. Normal tissues include The Cancer Genome Atlas (TCGA) paired normal samples and Genotype-Tissue Expression (GTX) normal samples (37). The UALCAN online database (http://ualcan.path.uab.edu) was used to analyze the expression levels of $H O X B s$ in different grades and stages of GC (38). A P value $<0.05$ was considered statistically significant.

\section{Bioinformatics analyses of patient survival data}

The Kaplan-Meier plotter GC database (http://kmplot. com/analysis/index.php? $\mathrm{p}=$ service \& cancer $=$ gastric) was used to analyze the quantity and expression of $H O X B s$, and overall survival (OS), progression-free survival (PFS), and post-progression survival (PPS) in patients with GC. Patients were allocated to high or low HOXB expression groups, and the survival analysis was performed using the Kaplan-Meier method. The hazard ratios (HRs) and 95\% confidence intervals (CIs) were calculated. The difference analysis was performed using the log-rank test.

\section{Bioinformatics analyses of the gene-gene interaction network}

The gene-gene interaction network of HOXBs was constructed with the GeneMANIA database (http://www. genemania.org). The GeneMANIA database is used to generate assumptions about gene function, analyze lists of genes, prioritize genes for functional analyses, and predict gene function (39). The GeneMANIA database uses extensive genomic and proteomic data to find genes with similar functions.

\section{Bioinformatics analyses of gene function and patbway}

The genes co-expressed with $H O X B 9$ were identified using the cBioPortal database (https://www.cbioportal.org/) (40). The data set for stomach adenocarcinoma from TCGA, which included 478 cases with pathology reports, was used to further analyze gene expression using the cBioPortal database. In addition, the cBioPortal database was used to obtain the correlation coefficients among $H O X B$ members and the gene sets co-expressed with HOXB9. We set the Spearman correlation coefficient $>0.4$ to explore genes that were significantly related to $H O B X$. The Database for Annotation Visualization and Integrated Discovery (DAVID) (https://david.ncifcrf.gov) was used to analyze the function and pathway of the genes co-expressed with HOXB9. A difference was considered statistically significant when the P value was $<0.05$ Gene Ontology (GO; http:// www.geneontology.org/) and Kyoto Encyclopedia of Genes and Genomes (KEGG; http://www.genome.jp/ 
kegg/pathway.html) pathway enrichment analyses were subsequently conducted to identify genes and pathways at the biologically functional level.

\section{Sample sources and clinical data}

A total of 70 effective GC tissue samples were collected from GC patients who underwent surgery at The First Affiliated Hospital of Jinzhou Medical University from 2010 to 2014. All the samples were embedded in paraffin. No patients received radiotherapy, chemotherapy, or hormone therapy before surgery. All the pathological sections were evaluated by pathologists and the diagnoses were clear. We identified 21 cases of tubular adenocarcinoma, 15 cases of papillary adenocarcinoma, 19 cases of mucinous adenocarcinoma, 9 cases of signet ring cell carcinoma, and 6 cases of undifferentiated carcinoma.

\section{Immunohistochemical analysis}

Paraffin sections of the GC tissues were made every $5 \mu \mathrm{m}$. The expression of HOXB9 was detected using the streptavidin-peroxidase method. The gastric tissue sections with $H O X B 9$ expression were positive, and phosphate buffer was used as the negative control. We used HOXB9 polyclonal antibody (Abcam, Cambridge, UK; 1:75) to explore the patient's tissue expression. The following scores were assigned according to the dyeing intensity: no pigment (0 points), light yellow (1 point), brown yellow (2 points), and dark brown (3 points). The following scores were assigned according to the percentage of stained cells: $<5 \%$ (0 points), $5-25 \%$ (1 point), $26-50 \%$ ( 2 points), $51-75 \%$ ( 3 points), and $>75 \%$ ( 4 points). The staining intensity was multiplied by the proportion of the stained cells. A score of 3-5 points indicated low expression, and a score of 6-12 points indicated high expression. Two professionals read the film independently.

\section{Statistical analysis}

The relationship between the expression of $H O X B 9$ protein and the clinicopathological features was analyzed by a nonparametric test. The Mann-Whitney $U$ test was used to compare two groups, and the Kruskal-Wallis test was used to compare multiple groups. A Cox proportional hazards model was used for the multivariate analysis. A P value $<0.05$ indicated a statistically significant difference.

\section{Results}

\section{Transcriptional levels of HOXBs in patients with stomach cancer}

First, we used the Oncomine database to examine the transcriptional levels of HOXBs in GC and normal tissues (see Figure 1). Most cancer types had significantly increased expressions of 1 or more $H O X B$ family members (see Figure 1). Multiple $H O X B s$, including $H O X B 3, H O X B 5$, HOXB6, HOXB7, HOXB9, and HOXB13, were more upregulated in GC samples than normal tissue samples in different publications (41-44). For example, D'Errico et al. (41) and Cho et al. (42) reported that the expression of $H O X B 3, H O X B 5, H O X B 6, H O X B 7$, and $H O X B 9$ was more than 2 -fold higher in gastric intestinal-type adenocarcinoma tissue samples than normal tissue samples (see Table 1). Additionally, in the gastric statistics data of Wang et al. (43), Cho et al. (42), and D'Errico et al. (41), the expression of $\mathrm{HOXB7}$ was elevated (2- to 5-fold) in GC samples in 6 paired comparisons. According to the Cui gastric statistics data, the expression of HOXB9 was 39fold higher in GC samples than normal tissue samples (see Table 1).

\section{Relationship between the messenger RNA (mRNA) levels of HOXBs and the clinicopathological parameters of patients with $G C$}

Using the GEPIA data set, we compared the mRNA expression of HOXB factors between GC and adjacent normal tissues. The results indicated that the expression levels of $H O X B 5$, HOXB6, HOXB7, HOXB9, and HOXB13 were significantly higher in GC tissues than normal tissues (see Figure 2A,2B). We then used the UALCAN online analysis tool to examine the relationship between the mRNA expression level of $H O X B$ family members and cancer stage and tumor grade in GC patients. As Figure $2 C$ shows, the mRNA levels of $H O X B$ family members were highly correlated with patients' individual cancer stages, and the tumors of patients with more advanced cancer stages tended to have higher $H O X B$ mRNA levels. Compared to normal tissues, tumor tissues from patients in stage 4 had the highest mRNA expressions of HOXB1 and HOXB9 (see Figure 2C), while tumor tissues from patients in stages 2 and 3 had the highest mRNA expressions of $H O X B 3, H O X B 5$, HOXB6, HOXB7, HOXB8, and HOXB9 (see Figure 2C). 
HOXB1 HOXB2 HOXB3 HOXB4 HOXB5 HOXB6 HOXB7 HOXB8 HOXB9 HOXB13

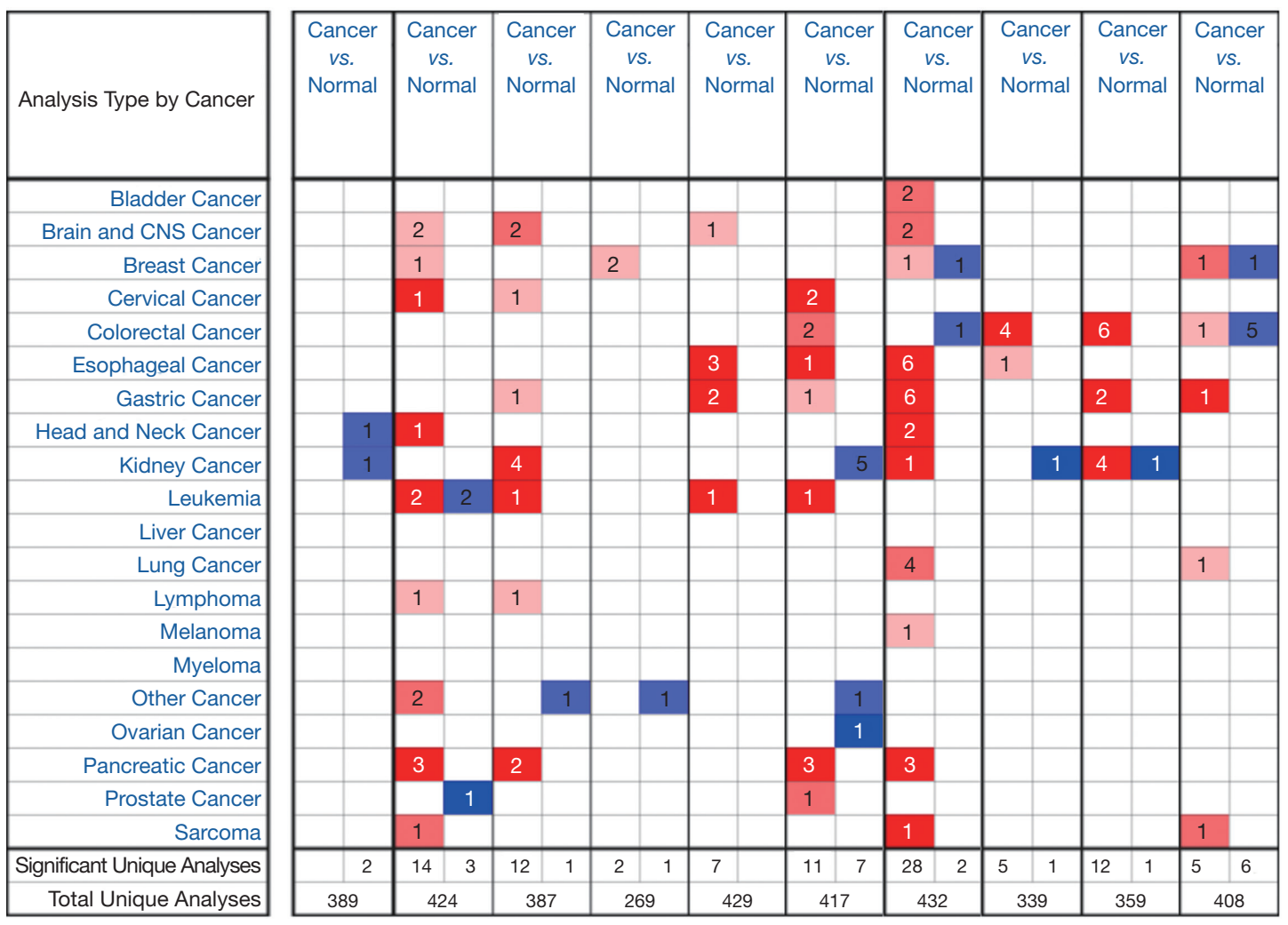

\section{$+15101051$}

Figure 1 The transcription levels of $H O X B$ factors in different types of cancers based on an analysis of the Oncomine database. The expression levels of HOXB1, HOXB2, HOXB3, HOXB4, HOXB5, HOXB6, HOXB7, HOXB8, HOXB9, and HOXB13 in different types of cancers were analyzed using the Oncomine database. Cancer types are listed on the left, and the numbers of significant unique analyses, and total analyses are listed at the bottom. Red boxes and blue boxes indicate upregulated and downregulated expressions in cancer samples vs. normal tissue samples, respectively. The color intensity represents the extend of the differentiated expression, and darker colors indicate more differentiated expression. The values in the red and blue boxes indicate the numbers of data sources. HOXB, Homeobox B.

Similarly, as Figure 2D shows, the mRNA expression levels of $H O X B$ family members were significantly correlated to tumor grades, and the mRNA levels of the $H O X B$ s tended to be higher in higher grade tumor samples. Compared to normal tissues, tumor tissues of patients at grade 3 had the highest mRNA levels of HOXB6, HOXB7, HOXB9, and HOXB13 (see Figure 2D), while the tumor tissues of patients at grade 2 had the highest mRNA expressions of HOXB4, HOXB6, HOXB7, HOXB9, and $H O X B 13$ (see Figure 2D). In addition, comparisons of $H O X B$ expressions at different tumor grades revealed that the mRNA levels of HOXB1, HOXB8, and HOXB9 from tumor tissues at grade 1 and 2 were obviously different. Taken together, these results indicate that mRNA expressions of $H O X B$ family members are significantly associated with the clinicopathological parameters of GC patients.

\section{The prognostic values of $\mathrm{HOXB}$ factors in $G C$}

We further analyzed the effects of $H O X B$ transcription levels on the survival of GC patients. The Kaplan-Meier plotter database was used to analyze correlations between the transcription levels of the mRNA of HOXBs in tumor tissues and the survival time of GC patients. The results revealed that the expressions of all the $H O X B$ factors were 
Table 1 The significant changes of $H O X B$ expression in transcription levels between different types of stomach cancer (Oncomine database)

\begin{tabular}{|c|c|c|c|c|c|}
\hline Gene ID & Types of stomach cancer vs. normal & Fold change & $P$ value & $t$-test & References \\
\hline HOXB2 & NA & NA & NA & NA & NA \\
\hline HOXBЗ & Gastric intestinal type adenocarcinoma vs. normal & 2.481 & $3.28 \mathrm{E}-8$ & 6.977 & D'Errico gastric statistics (41) \\
\hline HOXB4 & NA & NA & NA & NA & NA \\
\hline HOXB5 & Diffuse gastric adenocarcinoma vs. normal & 2.514 & $7.22 \mathrm{E}-7$ & 5.585 & Cho gastric statistics (42) \\
\hline HOXB6 & Gastric intestinal type adenocarcinoma vs. normal & 3.278 & $3.43 E-8$ & 6.298 & D'Errico gastric statistics (41) \\
\hline \multirow[t]{4}{*}{ HOXB7 } & GC vs. normal & 4.894 & $9.08 \mathrm{E}-6$ & 5.280 & Wang gastric statistics (43) \\
\hline & Gastric intestinal type adenocarcinoma vs. normal & 2.801 & $9.31 \mathrm{E}-7$ & 5.799 & Cho gastric statistics (42) \\
\hline & Gastric intestinal type adenocarcinoma vs. normal & 3.845 & $1.42 \mathrm{E}-11$ & 8.328 & D'Errico gastric statistics (41) \\
\hline & Gastric mixed adenocarcinoma vs. normal & 3.524 & $4.22 \mathrm{E}-5$ & 6.678 & D'Errico gastric statistics (41) \\
\hline HOXB8 & NA & NA & NA & NA & NA \\
\hline \multirow[t]{2}{*}{ HOXB9 } & GC vs. normal & 39.316 & 3.87E-11 & 7.045 & Cui gastric statistics (44) \\
\hline & Gastric intestinal type adenocarcinoma vs. normal & 2.305 & $1.53 \mathrm{E}-11$ & 8.333 & D'Errico gastric statistics (41) \\
\hline HOXB13 & GC vs. normal & 27.758 & $4.25 \mathrm{E}-8$ & 5.627 & Cui gastric statistics (44) \\
\hline
\end{tabular}

HOXB, Homeobox B; NA, not available; GC, gastric cancer.

significantly $(\mathrm{P}<0.05)$ associated with the OS, PFS, and PPS of patients with GC (see Figure 3). In particular, higher expressions of $H O X B 1, H O X B 2, H O X B 3, H O X B 4, H O X B 5$, $H O X B 6$, and $H O X B 9$ were associated with worse OS in GC patients (see Figure $3 A$ ), and higher expressions of HOXB1, HOXB2, HOXB3, HOXB4, HOXB5, HOXB6, and $H O X B 9$ were associated with worse PFS and PPS in GC (see Figure 3B,3C). Collectively, these results indicate that mRNA expressions of $H O X B$ family members in tumors are significantly associated with the survival of GC patients, and $H O X B$ factors are potentially useful biomarkers for predicting the prognosis of GC patients.

\section{Correlation and gene-interaction networks among $\mathrm{HOX}$ members}

We used GC transcriptomic data from the cBioPortal database (TCGA, Firehose Legacy) to analyze the mRNA transcription levels of $H O X B$ genes and determine the Spearman correlation coefficients among HOXBs members. Our analysis of the Spearman correlation coefficients suggested that many $H O X B$ factors, such as $H O X B 5$, HOXB6, HOXB9, HOXB8, and $H O X B 3$, had significant positive correlations with each other. In addition, the expressions of $H O X B 8$ or $H O X B 9$ were significantly correlated with the expressions $H O X B 3, H O X B 5, H O X B 6$, and HOXB7 (see Figure 4A).

Using the GeneMANIA online analysis tool, $H O X B$ family members were located at the central node, and 20 related nodes were selected outside the circle (see Figure 4B). These gene nodes were closely related to the HOXBs in terms of inter-gene interactions, co-expressions, predictions, co-localizations, and genetic interactions. The closely related genes included Src kinase associated phosphoprotein 1 (SKAP1), Homeobox A7 (HOXA7), Homeobox A10 (HOXA10), Homeobox A5 (HOXA5), and Homeobox C6 (HOXC6). In addition, the functional analysis of these proteins showed that they were closely related to the negative regulation of myeloid cell differentiation (false discovery rate $=1.21 \mathrm{E}-4$ ). Additionally, these proteins were associated with the regulation of myeloid cell differentiation, blood vessel morphogenesis, myeloid cell differentiation, 
A
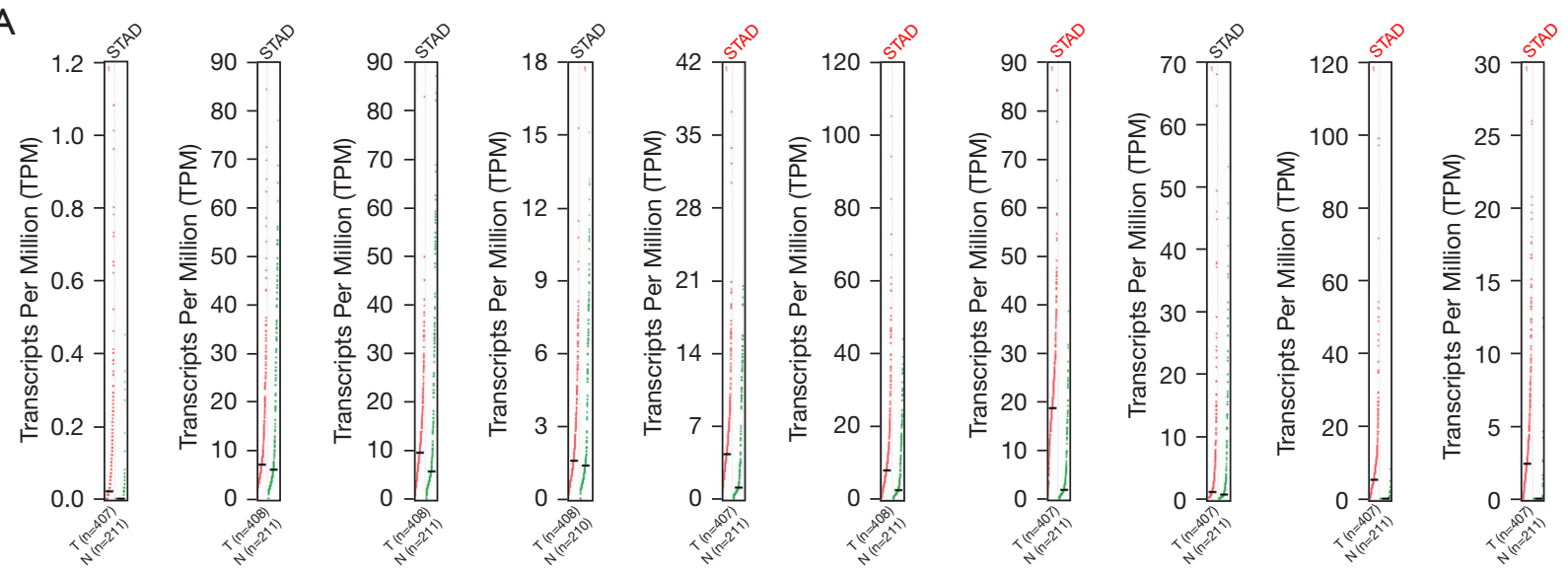

B
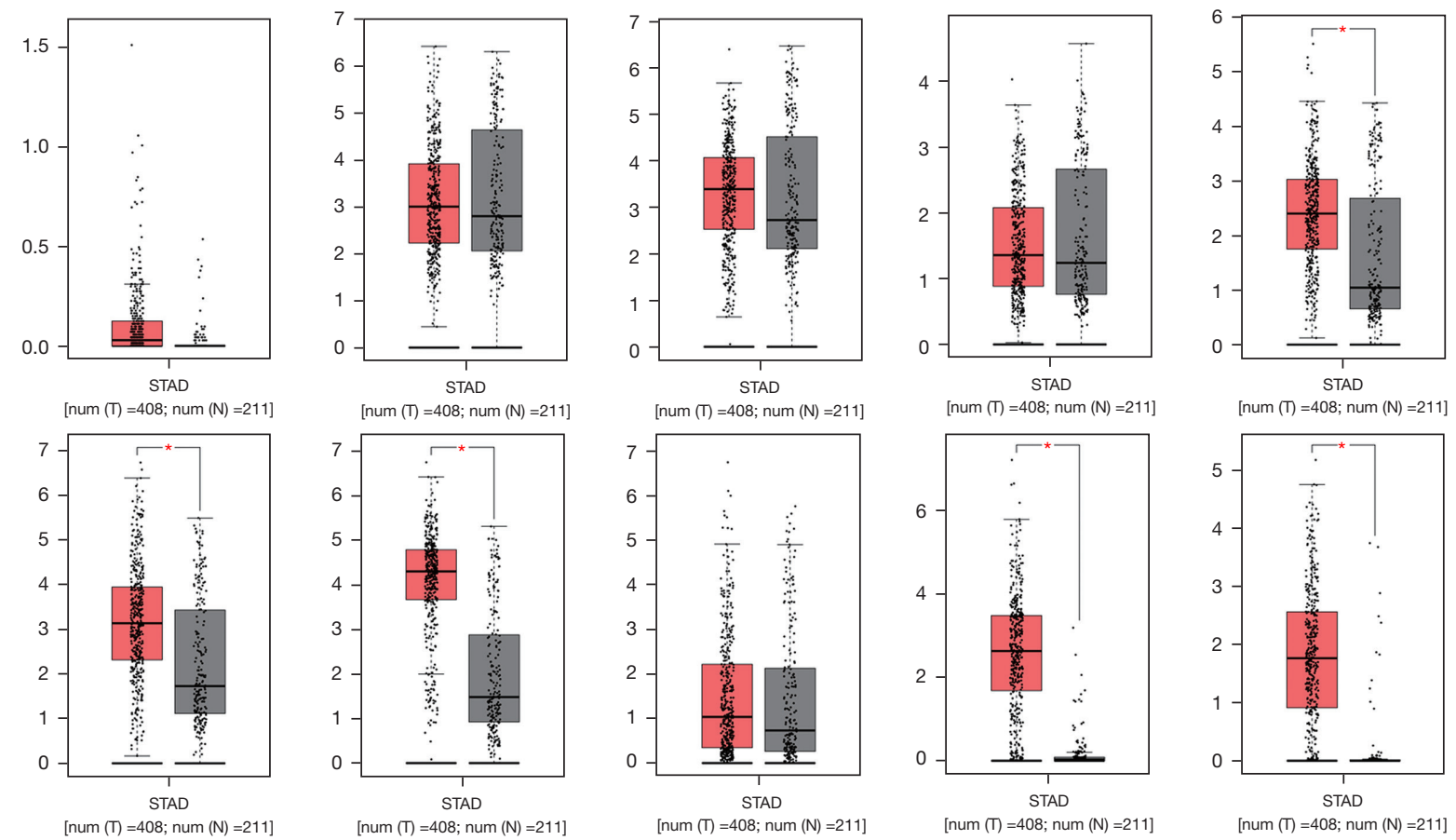
C
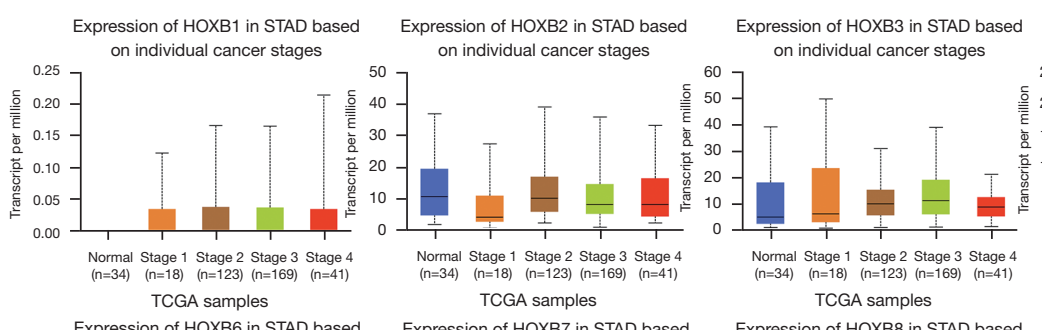

Expression of HOXB6 in STAD based Expression of HOXB7 in STAD based
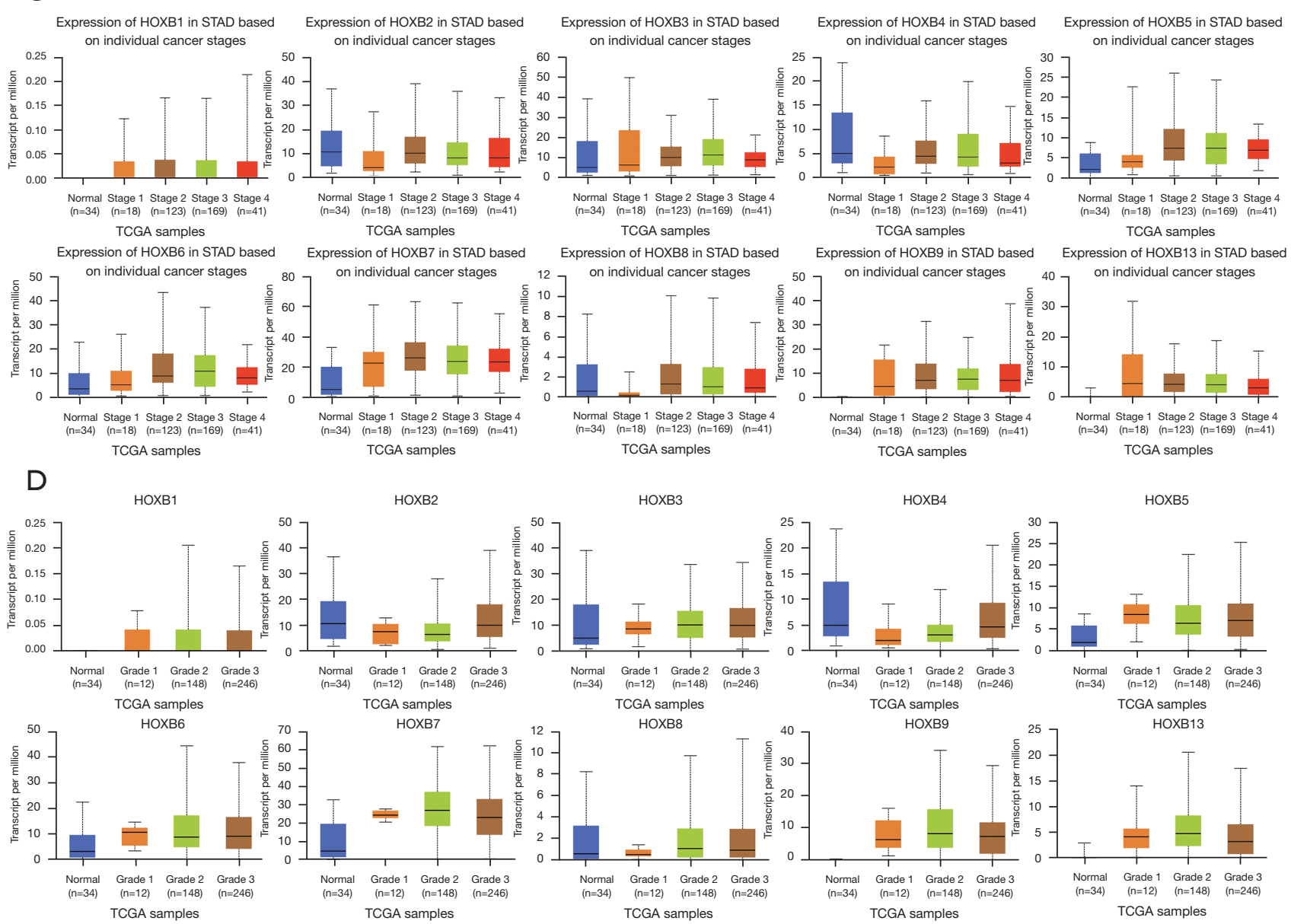

Figure 2 The expressions of HOXBs in GC samples and their correlation with tumor stages and grades. $(\mathrm{A}, \mathrm{B})$ The expression levels of HOXBs in gastric tumor samples and normal tissue samples were analyzed using the GEPIA data set. The data are presented in scatter diagrams (A) and box plots (B). (C,D) The correlations between HOXB expression levels and tumor stage (C) or tumor grade (D) of GC patients were analyzed using the UALCAN platform. *, P<0.05. HOXB, Homeobox B; GC, gastric cancer; GEPIA, Gene Expression Profiling Interactive Analysis.

blood vessel development, angiogenesis, and the negative regulation of cell differentiation (see Figure 4B).

\section{Functional and patbway enrichment analyses of $\mathrm{HOXB9}$}

We believe that HOXB9 can be used as a marker for the prognosis of GC. To further explore the biological function of HOXB9, the DAVID was used for the functional enrichment analysis. The KEGG pathway analysis results (see Figure $5 A$ ) showed that the main related pathways of the genes co-expressed with $H O X B 9$ were cell cycle, DNA replication, RNA transport, ribosome biogenesis in eukaryotes, homologous recombination, the Fanconi anemia pathway, and spliceosome, which are closely related to the occurrence and development of tumors.

The results of the GO enrichment analysis of biological processes showed that DNA replication, chromosome segregation, sister chromatid segregation, DNA conformation change, mitotic sister chromatid segregation, nuclear chromosome segregation, mitotic nuclear division, organelle fission, and DNA-dependent replication were regulated by $H O X B 9$ transcription in GC tissues (see Figure $5 B$ ). The results of the GO enrichment analysis of the cellular components showed that the chromosomal region condensed chromosome, chromosome, centromeric region, kinetochore, nuclear chromosome part, condensed 
A os
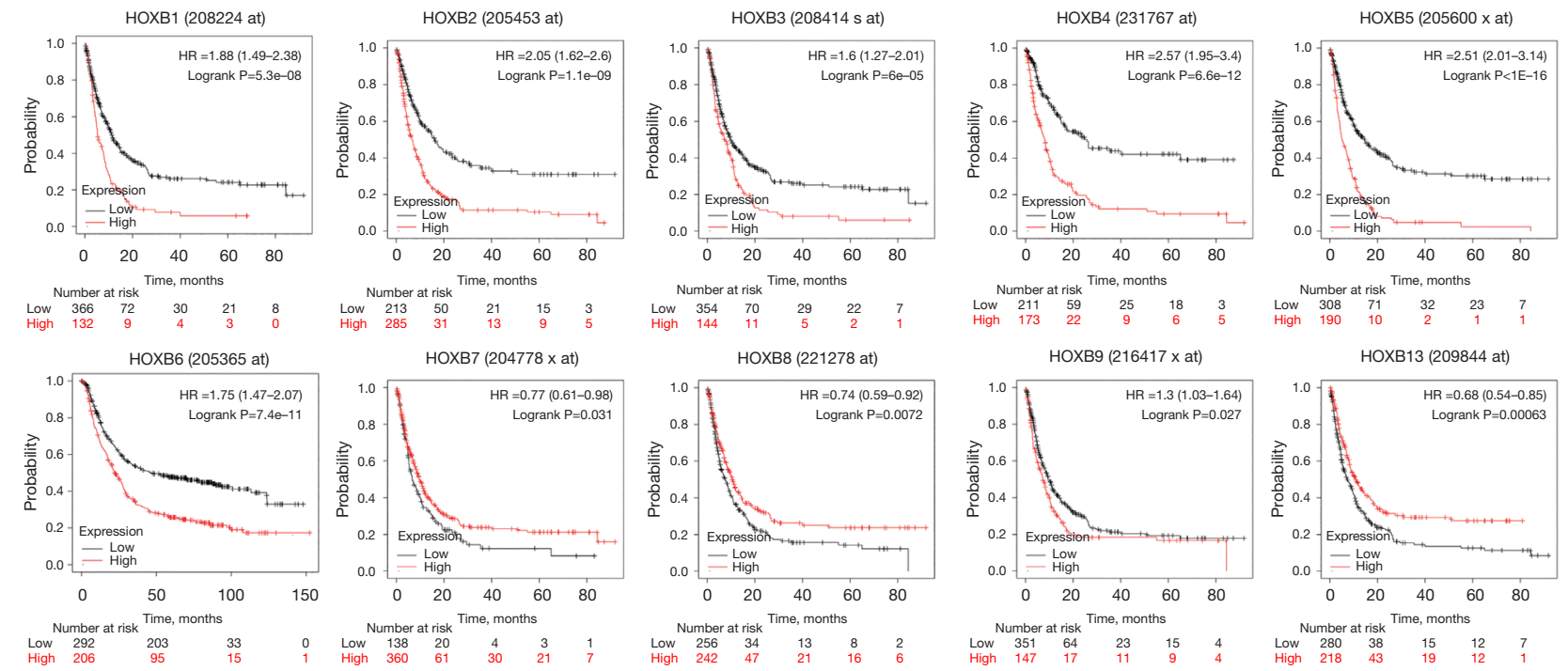

B ${ }^{\mathrm{FP}}$
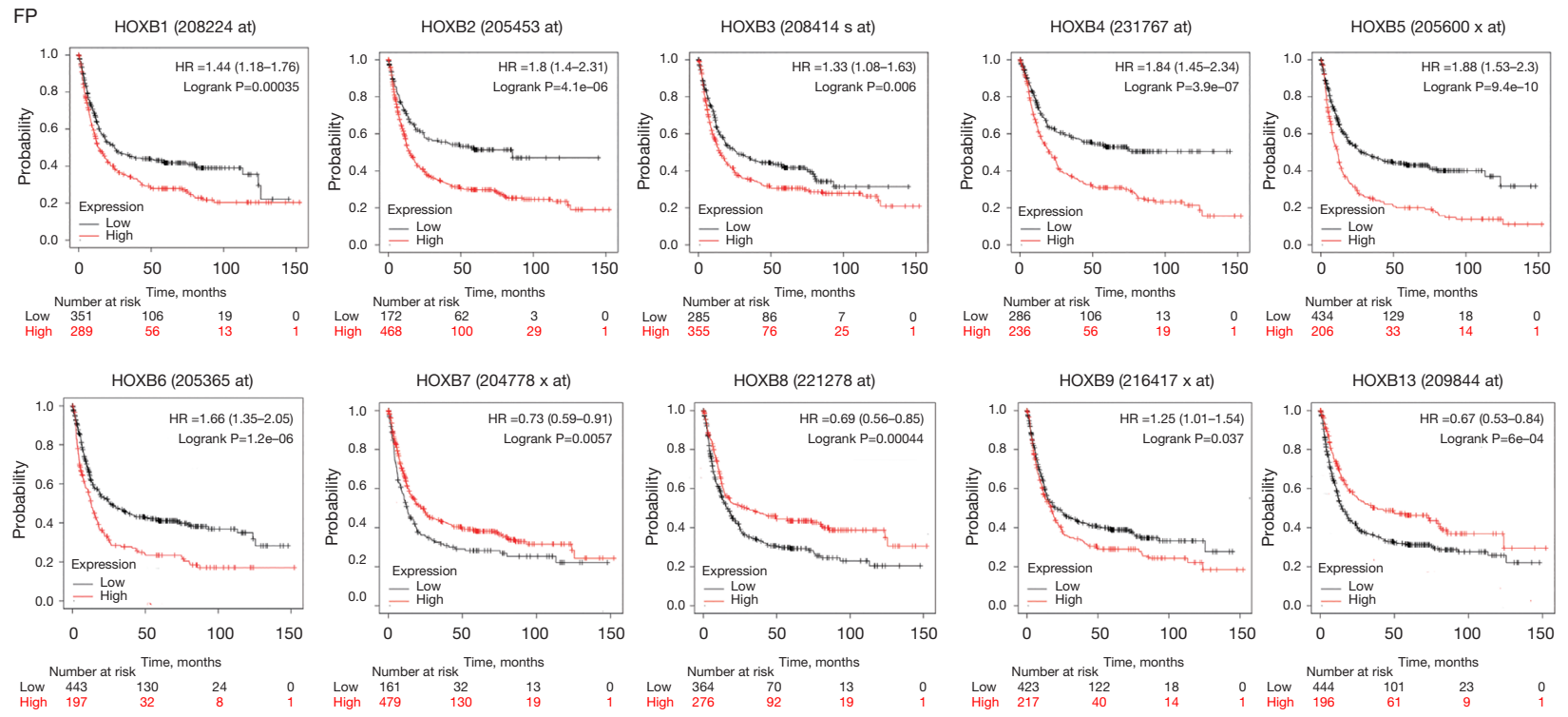

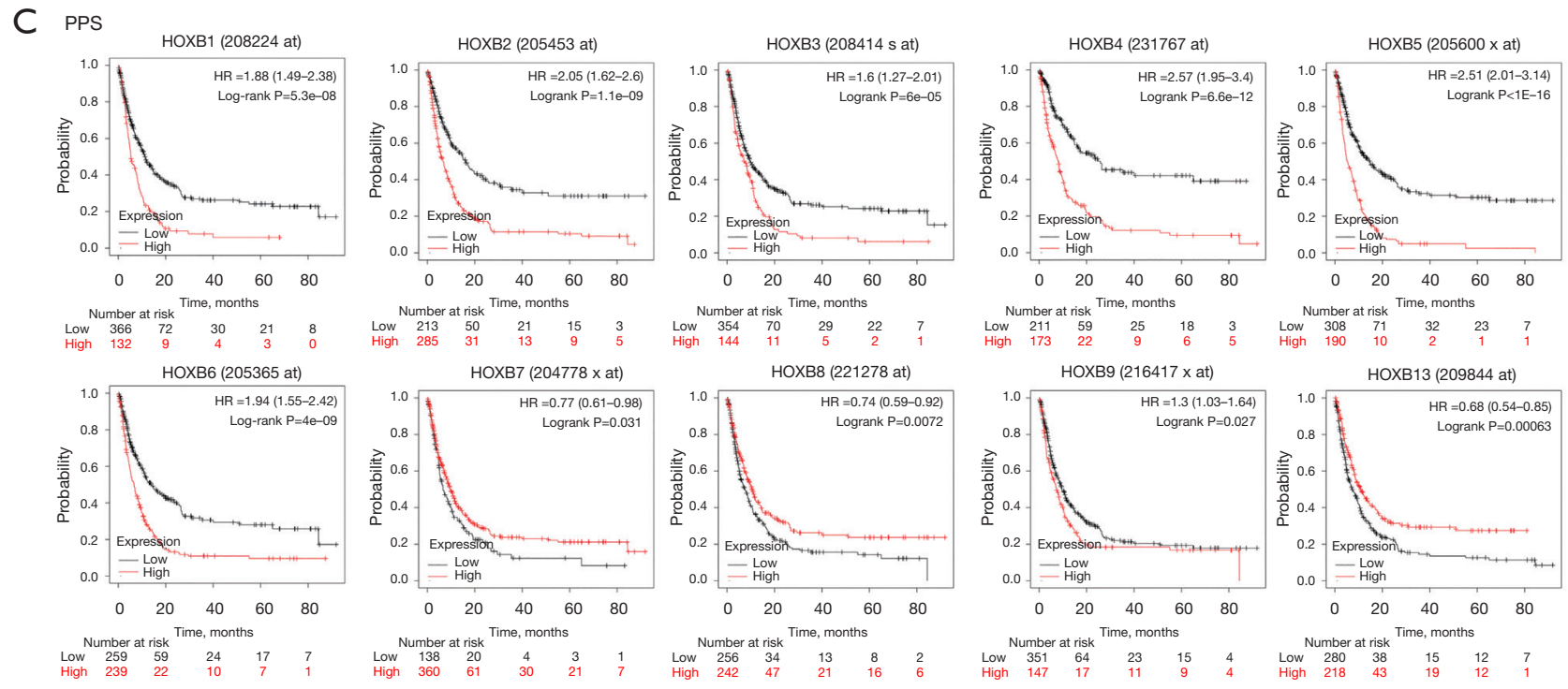

Figure 3 The prognostic value of the mRNA levels of HOXB factors in GC patients were explored using the Kaplan-Meier plotter database. The OS (A), PFS (B), and PPS (C) of patients with GC were analyzed by the Kaplan-Meier plotter database. The patients were grouped according to the expression levels of the indicated $H O X B$ factors in tumor. mRNA, messenger RNA; HOXB, Homeobox B; GC, gastric cancer; OS, overall survival; PFS, progression-free survival; PPS, post-progression survival; HR, hazard ratio.

chromosome kinetochore, spindle, chromatin, spindle pole were also significantly controlled by HOXB9 alterations (see Figure 5C). The results of the GO enrichment analysis of molecular functions showed that DNA-dependent adenosine triphosphatase (ATPase) activity, chromatin binding, helicase activity, DNA helicase activity, catalytic activity, acting on DNA, ATP-dependent DNA helicase activity, ATP-dependent helicase activity, purine NTPdependent helicase activity, ATPase activity, ATPase activity-coupled were also significantly controlled by the HOXB9 alterations (see Figure 5D).

\section{Higher levels of HOXB9 in GC were associated with higher stages and worse survival in a new cohort of patients}

To further validate the role of HOXB9 in GC progression, we investigated the association between $\mathrm{HOXB} 9$ expression in tumors, the stage, and survival in an independent new cohort of GC patients enrolled at our hospital. The cohort comprised 48 male patients and 22 female patients, and there was no significant difference in age between the two groups $(\mathrm{P}>0.05)$. According to the degree of HOXB9 immunohistochemical staining in gastric tissue sections of patients with GC, patients with GC were divided into two groups. There were significant differences between the groups with high and low expressions of $H O X B 9$ in relation to the degree of lymph node metastasis $(\mathrm{P}=0.006)$ and distant metastasis $(\mathrm{P}<0.001)$. However, HOXB9 expression level was not significantly correlated with sex, age, or tumor ( $\mathrm{T})$ stage $(\mathrm{P}>0.05$; see Table 2$)$.

Seventy patients with GC were selected for follow-up observation. The last follow-up date was March 10, 2019. Among these 70 patients, 53 patients (75.71\%) had complete follow-up information, and 17 lost patients (24.29\%) had incomplete follow-up information. The follow-up period for all patients commenced on the day of their operation, and continued to the deadline. The deadline for the lost patients was the date of their last visit. The survival analysis showed that the survival rate of patients with a high expression of HOXB9 was significantly lower than that of patients with a low expression of HOXB9 $(\mathrm{P}<0.0001$; see Figure $6 A)$. A Cox regression model was used to analyze the age, gender, tumor, lymph node metastasis, and distant metastasis of all patients with GC according to the expression of HOXB9. The results of the univariate Cox regression analysis showed that there was a significant correlation between OS and HOXB9 expression $(\mathrm{P}<0.001)$ and distant metastasis $(\mathrm{P}<0.001)$. The results of the multivariate Cox regression analysis showed that the high expression of HOXB9 $(\mathrm{P}=0.002)$ was an independent risk factor for the survival and prognosis of 
A

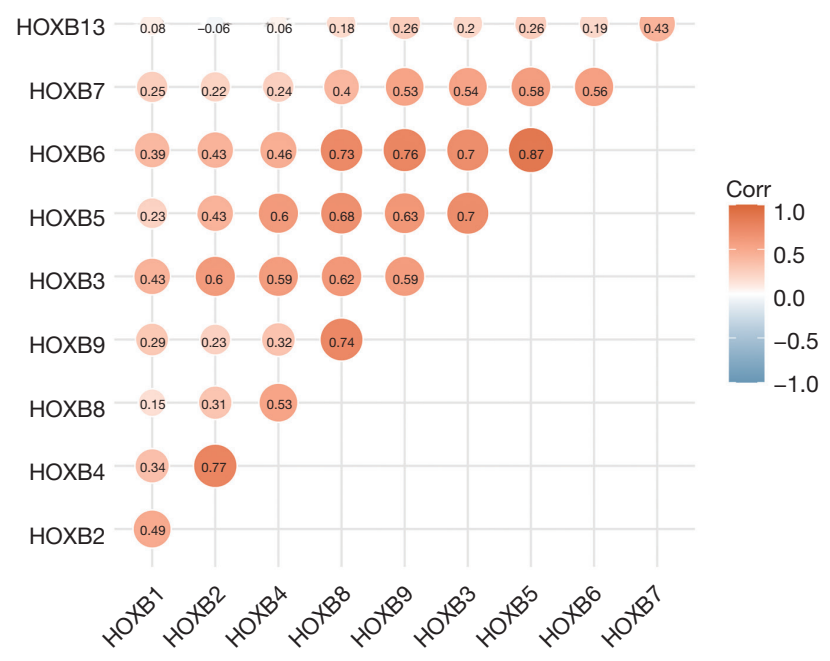

GeneMANIA report

Created on: 25 October 2020 19:05:26

Created on: 25 October 2020 19:05:26

Application version: 3.6 .0

B
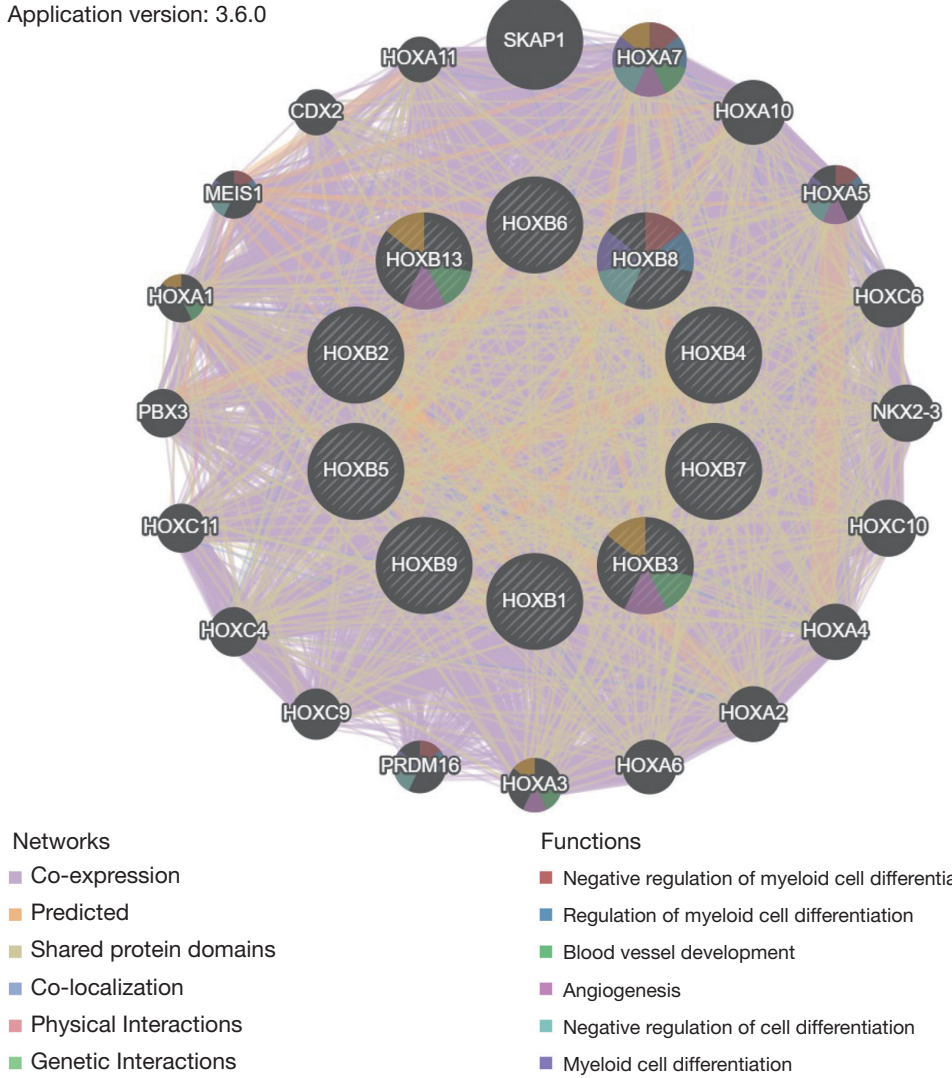

Functions

- Negative regulation of myeloid cell differentiation

- Regulation of myeloid cell differentiation

Blood vessel development

angiogenesis

negative regulation of cell differentiation

- Myeloid cell differentiation

Blood vessel morphogenesis

Figure 4 Correlation and gene interaction networks among $H O X B$ members. (A) The Spearman correction method was applied to the correlation coefficients among the HOXBs members. A correlation coefficient $>0.4$ indicates a strong correlation. Blue is negative; red is positive. The shade of the color indicates a change in the degree of correlation. (B) Gene-gene interaction networks among members of the HOXB family. The names of different genes are at the nodes, the radius of the nodes represents the intensity of the interaction, the color of the nodes represents the possible biological function of each gene, and the color of the line between nodes represents the type of interaction between the genes. HOXB, Homeobox B. 
A

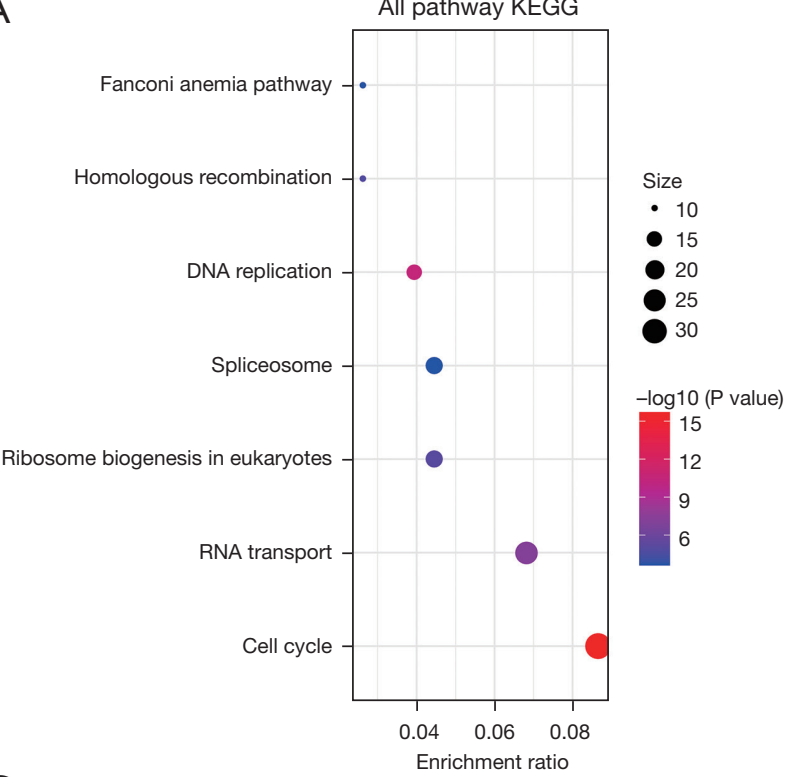

C

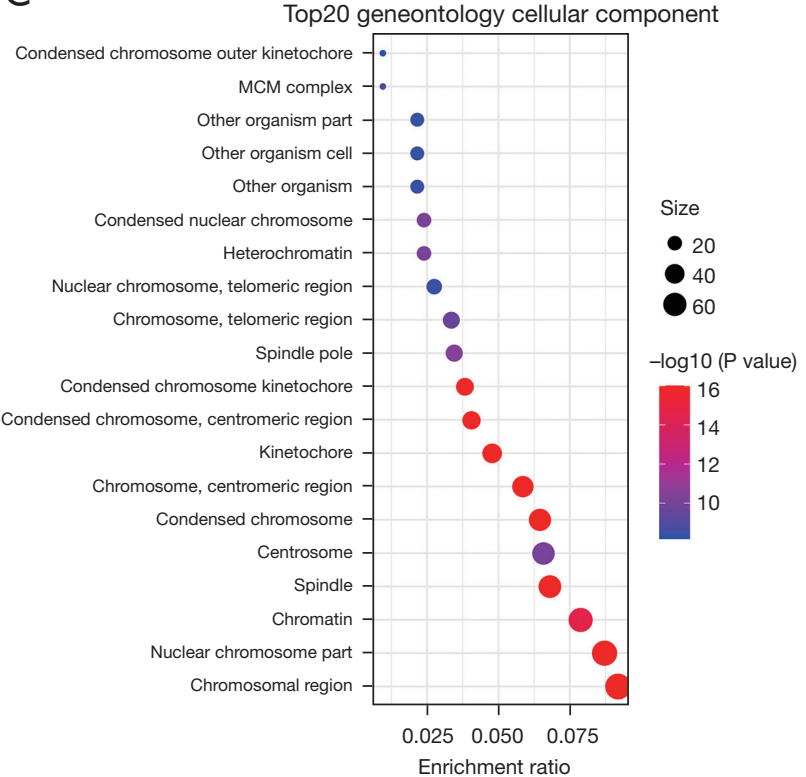

B

B Top20 geneontology biological process

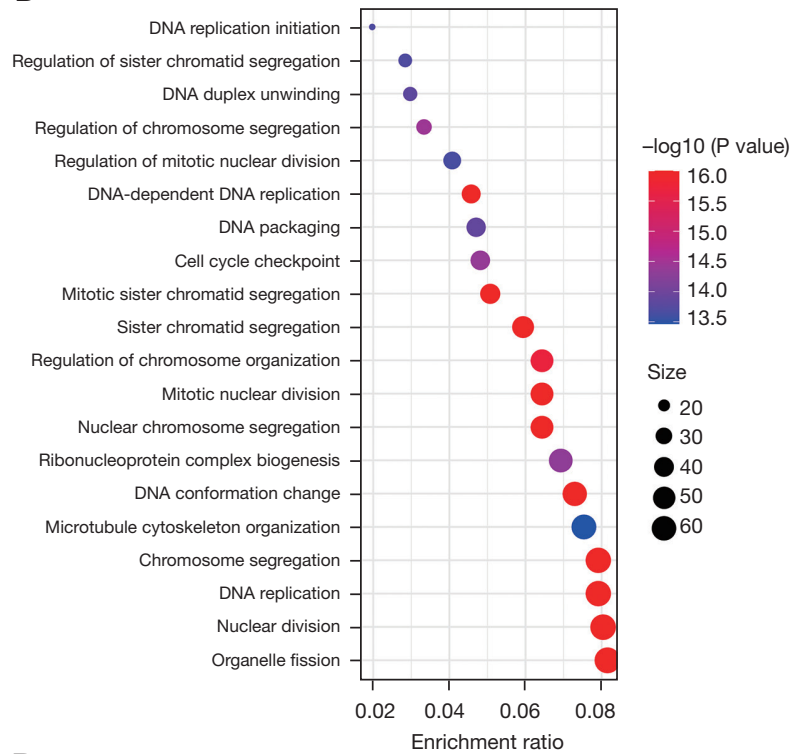

D

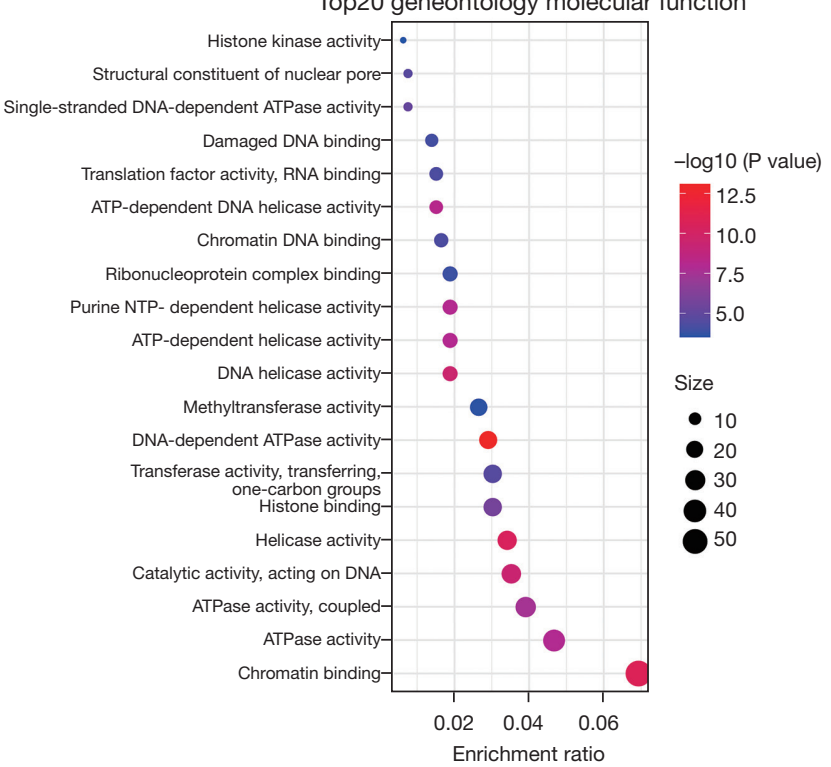

Figure 5 The function of $H O X B s$ and genes significantly associated with $H O X B 9$ alterations. (A) Gene function and pathway enrichment analysis of $H O X B 9$ co-expression. A Spearman correlation coefficient $>0.4$ was used as the selection criteria. Nine hundred and sixty genes co-expressed with $H O X B 9$ were obtained from the cBioPortal database. The bubble chart was constructed with the ggplot2.R software package. (B-D) GO pathway enrichment analyses were subsequently conducted to identify genes and pathways in terms of the biological processes (B), cellular components (C), and molecular functions (D). HOXB, Homeobox B; GO, Gene Ontology.

patients with GC (see Figure 6B).

\section{Immunobistochemical staining for $\mathrm{HOXB} 9$ and relation between HOXB9 expression and clinicopathologic factors}

HOXB9 expression in patients with GC. The expression of $H O X B 9$ was evaluated in 70 tumor specimens. Immunohistochemical staining for HOXB9 (Figure 7) was performed. Staining intensity was graded as none, weak and intense. According to relation between HOXB9 expression and clinicopathologic factors in 70 GC patients (Table 3), a total of 36 tumors $(51.4 \%)$ were positive for HOXB9 
Table 2 The expression of HOXB9 in GC is related to the clinical characteristics of patients with GC

\begin{tabular}{|c|c|c|c|c|}
\hline \multirow{2}{*}{ Characteristics } & \multirow{2}{*}{$\mathrm{N}$} & \multicolumn{2}{|c|}{ HOXB9 expression } & \multirow{2}{*}{$P$ value } \\
\hline & & Low (n) & High (n) & \\
\hline Age (years) & & & & 0.071 \\
\hline$<60$ & 27 & 16 & 11 & \\
\hline$\geq 60$ & 43 & 16 & 27 & \\
\hline Gender & & & & 0.288 \\
\hline Male & 48 & 24 & 24 & \\
\hline Female & 22 & 8 & 14 & \\
\hline T stage & & & & 0.187 \\
\hline $\mathrm{T} 1$ & 2 & 1 & 1 & \\
\hline T2 & 10 & 7 & 3 & \\
\hline T3 & 16 & 9 & 7 & \\
\hline T4 & 42 & 15 & 27 & \\
\hline \multicolumn{2}{|c|}{ Lymph node metastasis } & & & 0.006 \\
\hline No & 10 & 8 & 2 & \\
\hline $\mathrm{N} 1$ & 28 & 16 & 12 & \\
\hline N2 & 24 & 7 & 17 & \\
\hline N3 & 8 & 1 & 7 & \\
\hline Metastasis & & & & 0.000 \\
\hline No & 35 & 29 & 6 & \\
\hline Yes & 35 & 3 & 32 & \\
\hline
\end{tabular}

HOXB, Homeobox B; GC, gastric cancer.

expression. HOXB9 staining was observed in the nucleus of GC cells.

\section{Discussion}

In addition to cancer genetics, the abnormal regulation of epigenetics is also involved in the occurrence, development, and prognosis of GC. As an important part of the epigenetic regulatory complex, $H O X B$ family proteins are involved in the occurrence of many cancers, including GC $(8,21)$. Some members of the $H O X B$ family have been shown to play a key role in GC; however, the unique roles of $H O X B$ family members in GC require clarification. In the present study, we analyzed the expressions and prognostic values of multiple $H O X B$ family members in GC using large-scale published data sets, and validated the relationship between high levels of $H O X B 9$ expression and survival in a new cohort of patients with GC.

The results of our study showed that the mRNA expression levels of $H O X B 3, H O X B 5, H O X B 6, H O X B 7$, $H O X B 9$, and $H O X B 13$ were higher in tumor tissues than normal tissues, and the mRNA expression of $H O X B s$ was significantly associated with cancer stages and tumor grades in GC patients. Additionally, higher mRNA expression levels of $H O X B 1, H O X B 2, H O X B 3, H O X B 4, H O X B 5$, and $H O X B 9$ were significantly associated with shorter OS in GC patients, while higher mRNA expressions of $H O X B 6$, $H O X B 7, H O X B 8$, and $H O X B 13$ were significantly related to favorable OS in GC patients. Thus, $H O X B 1, H O X B 2$, $H O X B 3, H O X B 4, H O X B 5$, and $H O X B 9$ function more like oncogenes, while $H O X B 6, H O X B 7, H O X B 8$, and $H O X B 13$ are putative tumor suppressors of GC. We also analyzed the functions and pathways of the mutations in $H O X B s$ and their 20 frequently altered neighbor genes in GC patients, and found that the 5 genes most associated with the $H O X B$ family were SKAP1, HOXA7, HOXA10, HOXA5, and HOXC6. Further research on these $H O X B$-correlated genes in the progression of GC needs to be conducted to extend our understanding of the cellular and molecular mechanisms underlying the altered levels of these HOXBs in the pathogenesis of GC.

Our comprehensive analyses indicated that HOXB9 has the greatest correlation with the prognosis of GC patients, and is closely related to the tumorigenesis and development of GC. Thus, the HOXB9 gene was selected for a follow-up study to further explore the biological processes and related signaling pathways involved in its coexpressed genes. $H O X B 9$ was previously identified as an oncogenic factor in human breast cancer (25). The ability of HOXB9 to modulate damage responses may involve EMT induced by the transcription target transforming growth factor-beta (45). Additionally, the positive roles of HOXB9 in promoting cellular proliferation, angiogenesis, and the migration and invasion of PCa cells were recently reported (27). Lymph node metastasis and lymphatic invasion are related to lymphogenic metastasis, a specific phenomenon of GC progression (46). The experimental results of Kato et al. (47) showed that the lymphangiogenic factor VEGF-D increased in TMK-1 cells transfected with HOXB9 gene. HOXB9 gene was overexpressed in human GC TMK-1 cells. That was the first study suggesting that $H O X B 9$ promoted lymphangiogenesis. Therefore, we concluded that HOXB9 affects lymphangiogenesis of tumor cells in vitro by promoting the expression of 


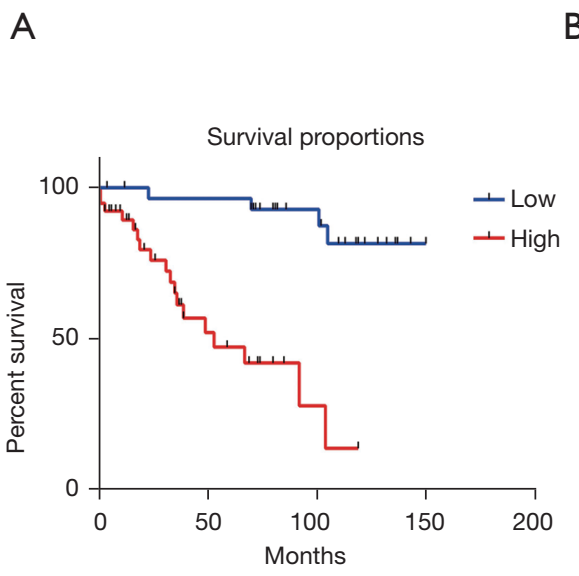

B

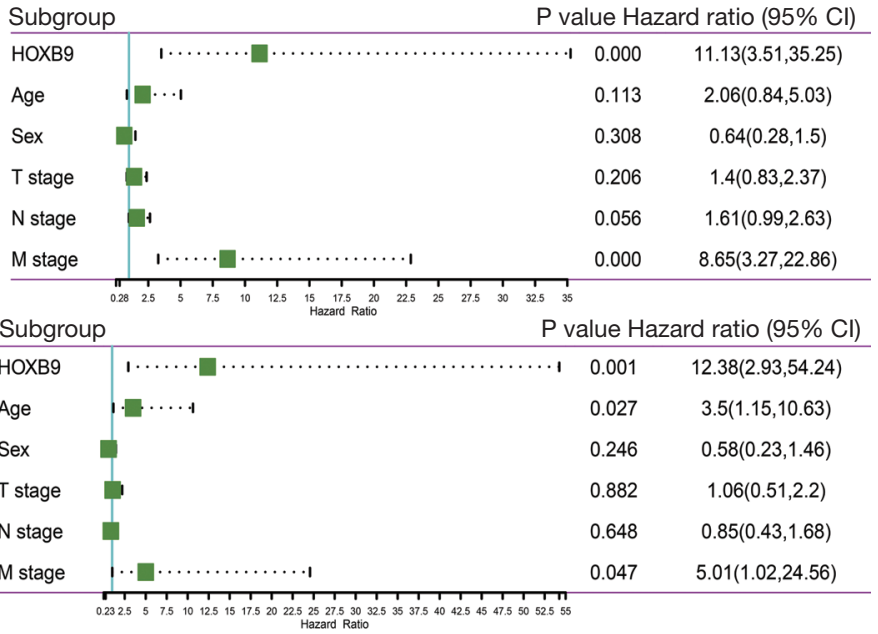

Figure 6 The expression of HOXB9 in GC and the survival of patients with GC and the factors affecting prognosis. (A) Relationship between the expression of HOXB9 in tumor tissues of patients with GC and patient survival. (B) Forest map of univariate and multivariate Cox regression analyses based on the survival data of patients with GC. HOXB, Homeobox B; GC, gastric cancer; CI, confidence interval.
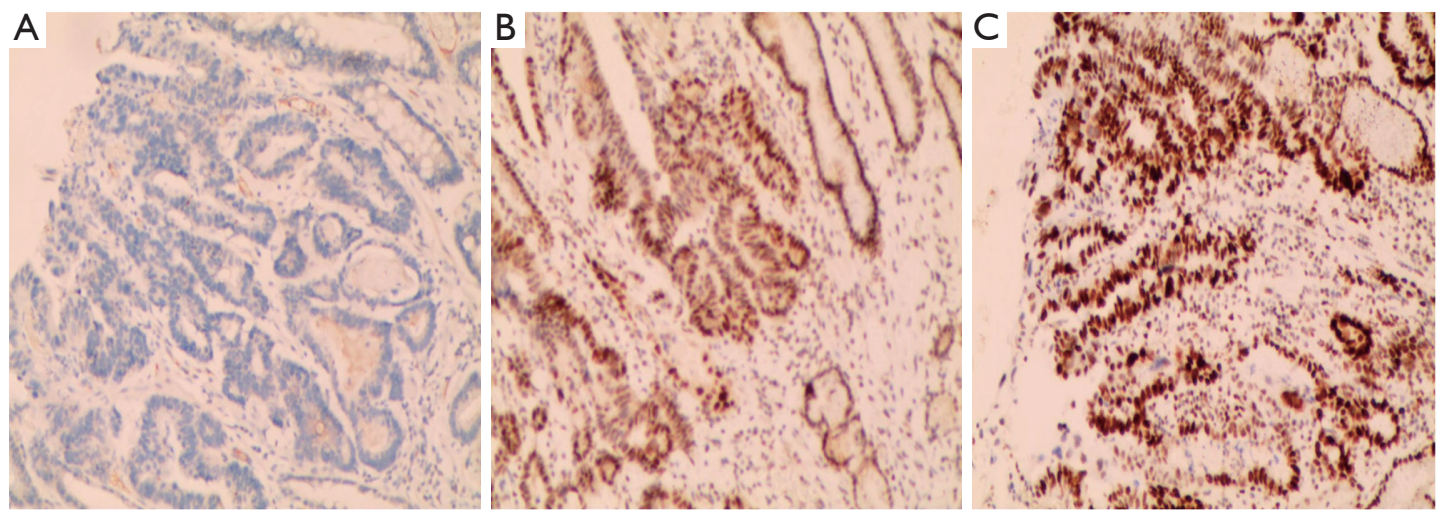

Figure 7 Immunohistochemical staining for homeobox B9 expression in human GC tissues. Staining intensity was graded as (A) no reaction, (B) weak reaction and $(C)$ intense reaction (original magnification, $\times 400$ ).

lymphangiogenic factor. HOXB9 expression was positively correlated with GC progression and lymphangiogenesis marker expression (44). Consistent with previous findings, our results also suggest that $H O X B 9$ plays an oncogenic role in the tumorigenesis of $\mathrm{GC}$, implying the conservative functions of $H O X B 9$ in human malignancies regardless of cancer types.

Various databases were used to further verify the correlation between HOXB9 expression and GC development. The Oncomine database showed that the expression level of $H O X B 9$ mRNA in GC was significantly upregulated. As the GC stage progressed, the expression level of $H O X B 9$ mRNA increased significantly. The results of the prognostic analysis showed that the upregulation of HOXB9 mRNA expression was most closely related to poor OS, PFS, and PPS in GC patients. We also analyzed the relationship between $H O X B 9$ expression and clinicopathological parameters, survival rate, and prognosis in GC patients. Patients with high expressions of $H O X B 9$ mostly had advanced GC and a poor prognosis. The Cox regression analysis showed that the high expression of $H O X B 9$ is an independent risk factor for survival and the prognosis of patients with GC. Thus, HOXB9 is a potential biomarker for early diagnosis, and can be used to evaluate the survival and prognosis of patients with GC.

The functions and pathways associated with $\mathrm{HOXB} 9$ 
Table 3 Relation between $H O X B 9$ expression and clinicopathologic factors in 70 GC patients

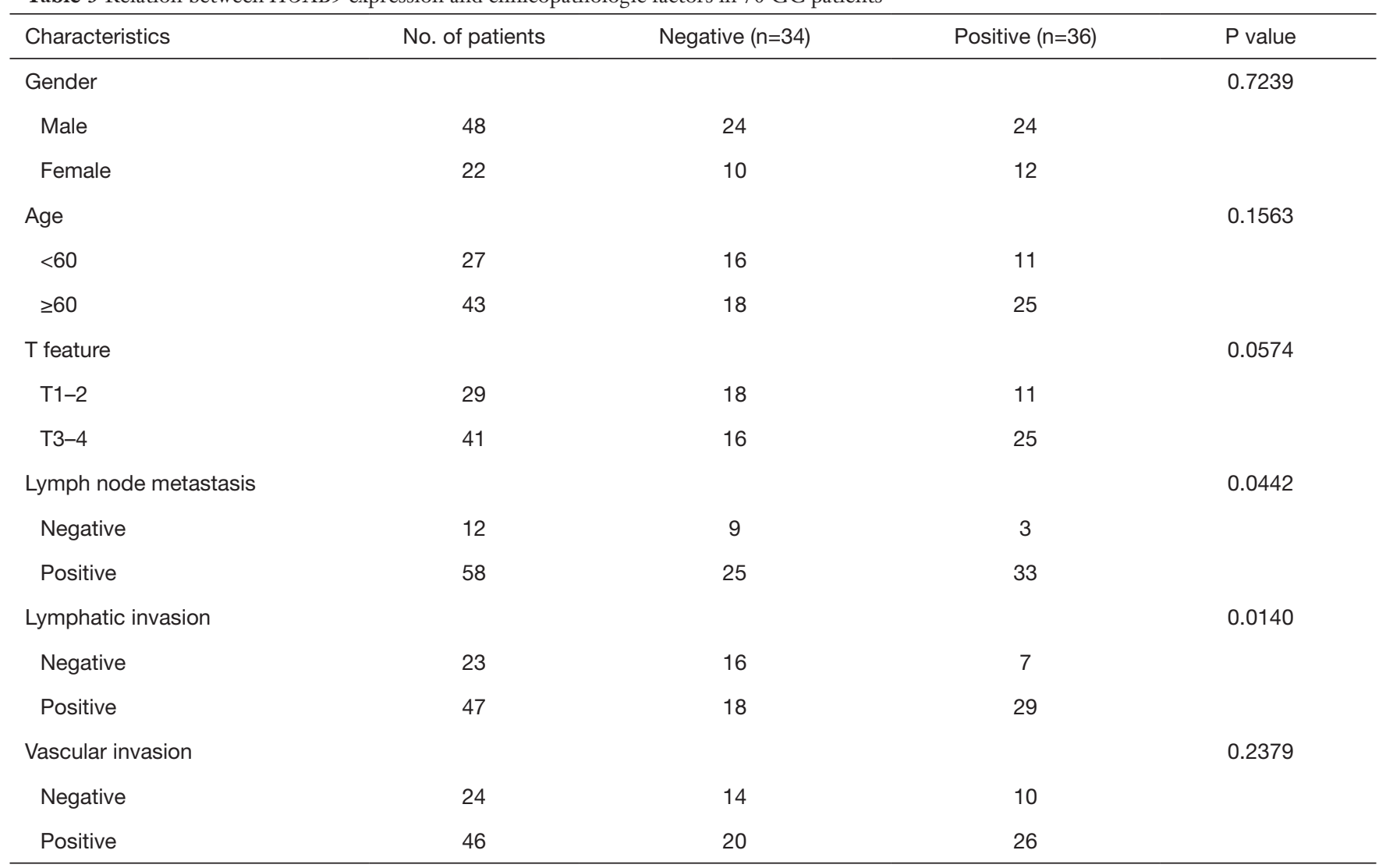

HOXB, Homeobox B; GC, gastric cancer; T, pathological depth of tumor invasion.

co-expressed genes that were identified using cBioPortal database were analyzed. The results showed that these pathways are involved in the regulation of DNA replication and dissociation. As $\mathrm{HOXB} 9$ has been reported to modulate the damage response in breast cancer cells (48), research needs to be conducted to determine whether HOXB9 modulates the irradiation-induced DNA damage responses of GC cells. In addition, it is reasonable to believe that HOXB9 and its co-expressed genes may promote the occurrence and development of GC via a complex regulatory network.

We used the GeneMANIA database to construct the interaction network between genes to further explore the mechanism of HOXBs in GC. The results showed that $H O X B$ s interacted closely with SKAP1, HOXA7, HOXA10, HOXA5, and HOXC6. SKAP2 and its related family member SKAP1 (mainly in T cells) have been shown to participate in cell adhesion by binding to integrin and actin (49). HOXA7 plays a key role in tumor invasion and metastasis by inducing EMT (50). HOXA10 aggravates GC by activating the fak1/STAT3 signaling pathway (51). The persistent endothelial expression of HOXA5 in vivo affects pathological angiogenesis and tumor progression (52). HOXC6 promotes tumor development through the indirect activation of the Notch and Wnt signaling pathways (53). Thus, genes interacting with $H O X B$ family members jointly promote the occurrence and development of tumors, and in-depth basic experiments need to be conducted to study the specific mechanism of action.

Our comprehensive analysis showed that $H O X B 9$ is highly expressed in GC tissues, and the high expression of $H O X B 9$ promotes the occurrence and development of $\mathrm{GC}$, and is positively correlated with the poor prognosis of GC patients. Thus, we believe that HOXB9 can be used as a novel biomarker for the clinical diagnosis, therapeutic treatment and prognosis of GC patients. At the same time, we further analyzed the clinicopathological and molecular characteristics of GC. The previous studies of Wang et al. showed that copy number gains (CNGs) at 20q11.2113.12 occurred frequently in hepatoid adenocarcinoma of 
the stomach (HAS), nearly 50\% of HAS tumors harbored at least one gene with a CNG at 20q11.21-13.12. This CNG tended to be related to more adverse biobehavioral, including poorer differentiation, greater vascular and nerve invasion, and greater liver metastasis. Pathway enrichment analysis revealed that the HIF-1 signaling pathway and signaling pathways regulating stem cell pluripotency were specifically enriched in HAS. The survival analysis showed that a preoperative serum alpha-fetoprotein (AFP) level $\geq 500 \mathrm{ng} / \mathrm{mL}$ was significantly associated with poorer OS $(\mathrm{P}=0.007)$ and tended to be associated with poorer diseasefree survival (DFS) ( $\mathrm{P}=0.05)$ (54). Previous study showed that $H O X B 9$ can promote adrenal tumor progression in a sex-dependent manner and have identified HOX factors as potential drug targets, leading to novel therapeutic approaches in adrenocortical carcinoma (ACC) (55). They identified $H O X B 9$ as a critical regulator of metastatic prostate cancer stem cells (mPCSC) behavior. This occurs through altering the expression of a panel of CSC growthand invasion/metastasis-related genes via TGF- $\beta$ signaling. Thus, targeting HOXB9 is a potential novel therapeutic PCa treatment strategy.

\section{Acknowledgments}

Funding: This work was supported by the Research Project of the National Natural Science Foundation of China (No. 81970358) and the Scientific Research Project of Liaoning Provincial Department of Education, Project No. JYTQN2020031.

\section{Footnote}

Reporting Checklist: The authors have completed the REMARK reporting checklist. Available at https://dx.doi. org/10.21037/jgo-21-598

Data Sharing Statement: Available at https://dx.doi. org/10.21037/jgo-21-598

Conflicts of Interest: All authors have completed the ICMJE uniform disclosure form (available at https://dx.doi. org/10.21037/jgo-21-598). The authors have no conflicts of interest to declare.

Ethical Statement: The authors are accountable for all aspects of the work in ensuring that questions related to the accuracy or integrity of any part of the work are appropriately investigated and resolved. All procedures performed in this study involving human participants were in accordance with the Declaration of Helsinki (as revised in 2013). The study was approved by Ethics Committee of The First Affiliated Hospital of Jinzhou Medical University (No. 1900034790) and informed consent was taken from all the patients.

Open Access Statement: This is an Open Access article distributed in accordance with the Creative Commons Attribution-NonCommercial-NoDerivs 4.0 International License (CC BY-NC-ND 4.0), which permits the noncommercial replication and distribution of the article with the strict proviso that no changes or edits are made and the original work is properly cited (including links to both the formal publication through the relevant DOI and the license). See: https://creativecommons.org/licenses/by-nc-nd/4.0/.

\section{References}

1. Bray F, Ferlay J, Soerjomataram I, et al. Global cancer statistics 2018: GLOBOCAN estimates of incidence and mortality worldwide for 36 cancers in 185 countries. CA Cancer J Clin 2018;68:394-424.

2. Luo M, Li L. Clinical utility of miniprobe endoscopic ultrasonography for prediction of invasion depth of early gastric cancer: a meta-analysis of diagnostic test from PRISMA guideline. Medicine (Baltimore) 2019;98:e14430.

3. Rugge M, Genta RM, Di Mario F, et al. Gastric cancer as preventable disease. Clin Gastroenterol Hepatol 2017;15:1833-43.

4. Siegel R, Ma J, Zou Z, et al. Cancer statistics, 2014. CA Cancer J Clin 2014;64:9-29.

5. Abate-Shen C. Deregulated homeobox gene expression in cancer: cause or consequence? Nat Rev Cancer 2002;2:777-85.

6. Scott MP. A rational nomenclature for vertebrate homeobox (HOX) genes. Nucleic Acids Res 1993;21:1687-8.

7. Paço A, de Bessa Garcia SA, Freitas R. Methylation in HOX Clusters and Its Applications in Cancer Therapy. Cells 2020;9:1613.

8. Xavier FC, Destro MF, Duarte CM, et al. Epigenetic repression of HOXB cluster in oral cancer cell lines. Arch Oral Biol 2014;59:783-9.

9. Vieux-Rochas M, Mascrez B, Krumlauf R, et al. Combined function of HoxA and HoxB clusters in neural crest cells. Dev Biol 2013;382:293-301. 
10. Han L, Liu D, Li Z, et al. HOXB1 is a tumor suppressor gene regulated by miR-3175 in glioma. PLoS One 2015;10:e0142387.

11. Cui F, Zhou Q, Xiao K, et al. The microRNA hsa-let$7 \mathrm{~g}$ promotes proliferation and inhibits apoptosis in lung cancer by targeting HOXB1. Yonsei Med J 2020;61:210-7.

12. Gonzalez-Herrera A, Salgado-Bernabe M, VelazquezVelazquez C, et al. Increased expression of HOXB2 and HOXB13 proteins is associated with HPV infection and cervical cancer progression. Asian Pac J Cancer Prev 2015;16:1349-53.

13. Segara D, Biankin AV, Kench JG, et al. Expression of HOXB2, a retinoic acid signaling target in pancreatic cancer and pancreatic intraepithelial neoplasia. Clin Cancer Res 2005;11:3587-96.

14. Li H, Zhu G, Xing Y, et al. miR-4324 functions as a tumor suppressor in colorectal cancer by targeting HOXB2. J Int Med Res 2020;48:300060519883731.

15. Chen X, Li LQ, Qiu X, et al. Long non-coding RNA HOXB-AS1 promotes proliferation, migration and invasion of glioblastoma cells via HOXB-AS1/miR-8853p/HOXB2 axis. Neoplasma 2019;66:386-96.

16. Niu M, Zhang N, Wang R, et al. MiR-340 is a biomarker for selecting treatment between chemotherapy and allogeneic transplantation in acute myeloid leukemia. Front Oncol 2019;9:1058.

17. Weiss FU, Marques IJ, Woltering JM, et al. Retinoic acid receptor antagonists inhibit miR-10a expression and block metastatic behavior of pancreatic cancer. Gastroenterology 2009; 137:2136-45.e1-7.

18. Teichweyde N, Kasperidus L, Carotta S, et al. HOXB4 promotes hemogenic endothelium formation without perturbing endothelial cell development. Stem Cell Reports 2018;10:875-89.

19. Huang X, Lee MR, Cooper S, et al. Activation of OCT4 enhances ex vivo expansion of human cord blood hematopoietic stem and progenitor cells by regulating HOXB4 expression. Leukemia 2016;30:144-53.

20. Winnik S, Klinkert M, Kurz H, et al. HoxB5 induces endothelial sprouting in vitro and modifies intussusceptive angiogenesis in vivo involving angiopoietin-2. Cardiovasc Res 2009;83:558-65.

21. Spencer DH, Young MA, Lamprecht TL, et al. Epigenomic analysis of the HOX gene loci reveals mechanisms that may control canonical expression patterns in AML and normal hematopoietic cells. Leukemia 2015;29:1279-89.

22. Errico MC, Jin K, Sukumar S, et al. The Widening Sphere of Influence of HOXB7 in Solid Tumors. Cancer Res 2016;76:2857-62.

23. Wang T, Lin F, Sun X, et al. HOXB8 enhances the proliferation and metastasis of colorectal cancer cells by promoting EMT via STAT3 activation. Cancer Cell Int 2019;19:3.

24. Zhang L, Wang Y, Zhang L, et al. LINC01006 promotes cell proliferation and metastasis in pancreatic cancer via miR-2682-5p/HOXB8 axis. Cancer Cell Int 2019;19:320.

25. Seki H, Hayashida T, Jinno H, et al. HOXB9 expression promoting tumor cell proliferation and angiogenesis is associated with clinical outcomes in breast cancer patients. Ann Surg Oncol 2012;19:1831-40.

26. Hoshino Y, Hayashida T, Hirata A, et al. Bevacizumab terminates homeobox B9-induced tumor proliferation by silencing microenvironmental communication. Mol Cancer 2014;13:102.

27. Xu H, Wu S, Shen X, et al. Silencing of HOXB9 suppresses cellular proliferation, angiogenesis, migration and invasion of prostate cancer cells. J Biosci 2020;45:40.

28. Jung C, Kim RS, Zhang H, et al. HOXB13 is downregulated in colorectal cancer to confer TCF4mediated transactivation. Br J Cancer 2005;92:2233-9.

29. Muthusamy V, Duraisamy S, Bradbury CM, et al. Epigenetic silencing of novel tumor suppressors in malignant melanoma. Cancer Res 2006;66:11187-93.

30. Tommasi S, Karm DL, Wu X, et al. Methylation of homeobox genes is a frequent and early epigenetic event in breast cancer. Breast Cancer Res 2009;11:R14.

31. Tomioka N, Morita K, Kobayashi N, et al. Array comparative genomic hybridization analysis revealed four genomic prognostic biomarkers for primary gastric cancers. Cancer Genet Cytogenet 2010;201:6-14.

32. Hong CS, Jeong O, Piao Z, et al. HOXB5 induces invasion and migration through direct transcriptional up-regulation of $\beta$-catenin in human gastric carcinoma. Biochem J 2015;472:393-403.

33. Ding WJ, Zhou M, Chen MM, et al. HOXB8 promotes tumor metastasis and the epithelial-mesenchymal transition via ZEB2 targets in gastric cancer. J Cancer Res Clin Oncol 2017;143:385-97.

34. Chang Q, Zhang L, He C, et al. HOXB9 induction of mesenchymal-to-epithelial transition in gastric carcinoma is negatively regulated by its hexapeptide motif. Oncotarget 2015;6:42838-53.

35. Sui BQ, Zhang CD, Liu JC, et al. HOXB13 expression and promoter methylation as a candidate biomarker in gastric cancer. Oncol Lett 2018;15:8833-40. 
36. Rhodes DR, Kalyana-Sundaram S, Mahavisno V, et al. Oncomine 3.0: genes, pathways, and networks in a collection of 18,000 cancer gene expression profiles. Neoplasia 2007;9:166-80.

37. Tang Z, Li C, Kang B, et al. GEPIA: a web server for cancer and normal gene expression profiling and interactive analyses. Nucleic Acids Res 2017;45:W98-W102.

38. Chandrashekar DS, Bashel B, Balasubramanya SAH, et al. UALCAN: A portal for facilitating tumor subgroup gene expression and survival analyses. Neoplasia 2017;19:649-58.

39. Warde-Farley D, Donaldson SL, Comes O, et al. The GeneMANIA prediction server: biological network integration for gene prioritization and predicting gene function. Nucleic Acids Res 2010;38:W214-20.

40. Gao J, Aksoy BA, Dogrusoz U, et al. Integrative analysis of complex cancer genomics and clinical profiles using the cBioPortal. Sci Signal 2013;6:pl1.

41. D'Errico M, de Rinaldis E, Blasi MF, et al. Genomewide expression profile of sporadic gastric cancers with microsatellite instability. Eur J Cancer 2009;45:461-9.

42. Cho JY, Lim JY, Cheong JH, et al. Gene expression signature-based prognostic risk score in gastric cancer. Clin Cancer Res 2011;17:1850-7.

43. Wang Q, Wen YG, Li DP, et al. Upregulated INHBA expression is associated with poor survival in gastric cancer. Med Oncol 2012;29:77-83.

44. Cui J, Chen Y, Chou WC, et al. An integrated transcriptomic and computational analysis for biomarker identification in gastric cancer. Nucleic Acids Res 2011;39:1197-207.

45. McCabe CD, Spyropoulos DD, Martin D, et al. Genomewide analysis of the homeobox C6 transcriptional network in prostate cancer. Cancer Res 2008;68:1988-96.

46. Pak KH, Jo A, Choi HJ, et al. The different role of intratumoral and peritumoral lymphangiogenesis in

Cite this article as: Li X, Chen S, Zhu Y, Fei J, Song L, Sun G, Niu W, Guo L, Wang J. Comprehensive bioinformatics analyses identified Homeobox B9 as a potential prognostic biomarker and therapeutic target for gastric cancer. J Gastrointest Oncol 2021;12(5):2132-2149. doi: 10.21037/jgo-21-598 gastric cancer progression and prognosis. BMC Cancer 2015;15:498.

47. Kato F, Wada N, Hayashida T, et al. Experimental and clinicopathological analysis of HOXB9 in gastric cancer. Oncol Lett 2019;17:3097-102.

48. Chiba N, Comaills V, Shiotani B, et al. Homeobox B9 induces epithelial-to-mesenchymal transition-associated radioresistance by accelerating DNA damage responses. Proc Natl Acad Sci U S A 2012;109:2760-5.

49. Togni M, Swanson KD, Reimann S, et al. Regulation of in vitro and in vivo immune functions by the cytosolic adaptor protein SKAP-HOM. Mol Cell Biol 2005;25:8052-63.

50. Tang B, Qi G, Sun X, et al. HOXA7 plays a critical role in metastasis of liver cancer associated with activation of Snail. Mol Cancer 2016;15:57.

51. Chen $\mathrm{W}, \mathrm{Wu} \mathrm{G}, \mathrm{Zhu} \mathrm{Y}$, et al. HOXA10 deteriorates gastric cancer through activating JAK1/STAT3 signaling pathway. Cancer Manag Res 2019;11:6625-35.

52. Cuevas I, Layman H, Coussens L, et al. Sustained endothelial expression of HoxA5 in vivo impairs pathological angiogenesis and tumor progression. PLoS One 2015;10:e0121720.

53. Kato F, Wada N, Hayashida T, et al. Experimental and clinicopathological analysis of HOXB9 in gastric cancer. Oncol Lett 2019;17:3097-102.

54. Wang Y, Sun L, Li Z, et al. Hepatoid adenocarcinoma of the stomach: a unique subgroup with distinct clinicopathological and molecular features. Gastric Cancer 2019;22:1183-92.

55. Francis JC, Gardiner JR, Renaud Y, et al. HOX genes promote cell proliferation and are potential therapeutic targets in adrenocortical tumours. Br J Cancer 2021;124:805-16.

(English Language Editor: L. Huleatt) 